\title{
TransMed: Transformers Advance Multi-Modal Medical Image Classification
}

\author{
Yin Dai ${ }^{1,2}$, Yifan Gao ${ }^{1}$ and Fayu Liu ${ }^{3, *}$ \\ 1 College of Medicine and Biological Information Engineering, Northeastern University, \\ Shenyang 110169, China; daiyin@bmie.neu.edu.cn (Y.D.); yifangao@stumail.neu.edu.cn (Y.G.) \\ 2 Engineering Center on Medical Imaging and Intelligent Analysis, Ministry Education, \\ Northeastern University, Shenyang 110169, China \\ 3 Department of Oromaxillofacial-Head and Neck Surgery, School of Stomatology, China Medical University, \\ Shenyang 110002, China \\ * Correspondence: 20031060@cmu.edu.cn
}

Citation: Dai, Y.; Gao, Y.; Liu, F. TransMed: Transformers Advance Multi-Modal Medical Image Classification. Diagnostics 2021, 11, 1384. https://doi.org/10.3390/ diagnostics11081384

Academic Editor: Sameer Antani

Received: 10 June 2021

Accepted: 28 July 2021

Published: 31 July 2021

Publisher's Note: MDPI stays neutral with regard to jurisdictional claims in published maps and institutional affiliations.

Copyright: (C) 2021 by the authors. Licensee MDPI, Basel, Switzerland. This article is an open access article distributed under the terms and conditions of the Creative Commons Attribution (CC BY) license (https:// creativecommons.org/licenses/by/ $4.0 /)$.

\begin{abstract}
Over the past decade, convolutional neural networks (CNN) have shown very competitive performance in medical image analysis tasks, such as disease classification, tumor segmentation, and lesion detection. CNN has great advantages in extracting local features of images. However, due to the locality of convolution operation, it cannot deal with long-range relationships well. Recently, transformers have been applied to computer vision and achieved remarkable success in large-scale datasets. Compared with natural images, multi-modal medical images have explicit and important long-range dependencies, and effective multi-modal fusion strategies can greatly improve the performance of deep models. This prompts us to study transformer-based structures and apply them to multi-modal medical images. Existing transformer-based network architectures require large-scale datasets to achieve better performance. However, medical imaging datasets are relatively small, which makes it difficult to apply pure transformers to medical image analysis. Therefore, we propose TransMed for multi-modal medical image classification. TransMed combines the advantages of CNN and transformer to efficiently extract low-level features of images and establish long-range dependencies between modalities. We evaluated our model on two datasets, parotid gland tumors classification and knee injury classification. Combining our contributions, we achieve an improvement of $10.1 \%$ and $1.9 \%$ in average accuracy, respectively, outperforming other state-of-the-art CNN-based models. The results of the proposed method are promising and have tremendous potential to be applied to a large number of medical image analysis tasks. To our best knowledge, this is the first work to apply transformers to multi-modal medical image classification.
\end{abstract}

Keywords: transformer; medical image classification; deep learning; multiparametric MRI; multi-modal

\section{Introduction}

Transformers were first applied in the field of natural language processing (NLP) [1]. It is a deep neural network mainly based on the self-attention mechanism to extract intrinsic features of textual data. Because of its powerful representation capabilities, researchers hope to find a way to apply transformers to computer vision tasks. Compared with text, images involve larger size, noise, and redundant modalities, so it is considered more difficult to use transformers on these tasks. Recently, transformers have made a breakthrough in computer vision. A large number of transformer-based methods have been proposed for computer vision tasks, such as DETR [2] for object detection, SETR [3] for semantic segmentation, ViT [4] and DeiT [5] for image classification.

Transformers have achieved success in natural images, but it has received little attention in medical image analysis, especially in multi-modal medical image fusion. Multimodal images are widely used in medical image analysis to achieve disease classification or lesion segmentation. The existing medical image multi-modal fusion based on deep 
learning can be divided into three categories: input-level fusion, feature-level fusion, and decision-level fusion [6]. Input-level fusion strategy fuses multi-modal images into the deep network by multi-channel, learns fusion feature representation, and then trains the network. Input-level fusion can retain the original image information to the maximum extent and learn the image features. Feature-level fusion strategy trains a single deep network by taking the image of each modality as a single input. Each representation is fused in the network layer, and the final result is fed to the decision layer to obtain the final result. Feature-level fusion network can effectively capture the information of different modalities of the same patient. Decision-level fusion integrates the output of each network to obtain the final result. Decision-level fusion network aims to learn more abundant information from different modalities independently.

However, they all have shortcomings in varying degrees. The input-level fusion strategy is difficult to establish the internal relationship between different modalities of the same patient, which leads to the degradation of the model performance. Each modality of the feature-level network corresponds to a neural network, which brings huge computational costs, especially in the case of a large number of modalities. The output of each modality of decision-level fusion is independent of each other, so the model cannot establish the internal relationship between different modalities of the same patient. In addition, like decision-level fusion strategy, decision-level fusion strategy is also computationally intensive.

Therefore, there is an urgent need to combine the three fusion strategies efficiently. A good multi-modal fusion strategy should achieve as much interaction between different modalities as possible with low computational complexity.

Compared with $\mathrm{CNN}$, transformers can effectively mine long-range relationships between sequences. The existing computer vision models based on transformer mainly deal with 2D natural images, such as ImageNet [7] and other large-scale datasets. The method of constructing sequences in 2D images is to cut the images into a series of patches. This kind of sequence construction method implicitly shows long-range dependencies, which is not very intuitive, so it may be difficult to bring significant performance improvement.

On the contrary, there are more explicit sequences in medical images, which contain important long-range dependency and semantic information, as shown in Figure 1. Due to the similarity of human organs, most visual representations are orderly in medical images. Destruction of these sequences will significantly reduce the performance of the model. It can be considered that compared with natural images, the sequence relationship of medical images (such as modality, slice, patch) holds more abundant information. In practice, clinicians will synthesize the pathological information of each modality to make the diagnosis. However, most of the existing multi-modal fusion methods do not or rarely consider the correlation of these sequences, and lack of modeling for these long-range dependencies. The transformer structure is an elegant, efficient, and powerful encoder for processing sequence relations, which is the motivation for us to propose the multi-modal medical image classification method based on transformers.

In this work, we present the first study to explore the tremendous potential of transformers in the context of multi-modal medical image classification. The proposed method is inspired by the property that the transformer is effective in extracting the relationship between sequences. However, due to the small scale of medical image datasets and the lack of sufficient information to establish the relationship between low-level semantic features, the performance of pure transformer networks based on ViT and DeiT is not satisfactory in multi-modal medical image classification. Therefore, we propose TransMed, which combines the advantages of $\mathrm{CNN}$ and transformer to capture low-level features and cross-modality high-level information. TransMed first processes the multi-modal images as sequences and sends them to $\mathrm{CNN}$, then uses transformers to learn the relationship between the sequences and make predictions. Since the transformer effectively models the global features of multi-modal images, TransMed outperforms the existing multi-modal 
fusion methods in terms of parameters, operation speed, and accuracy. A large number of experiments have proved the effectiveness of our method.

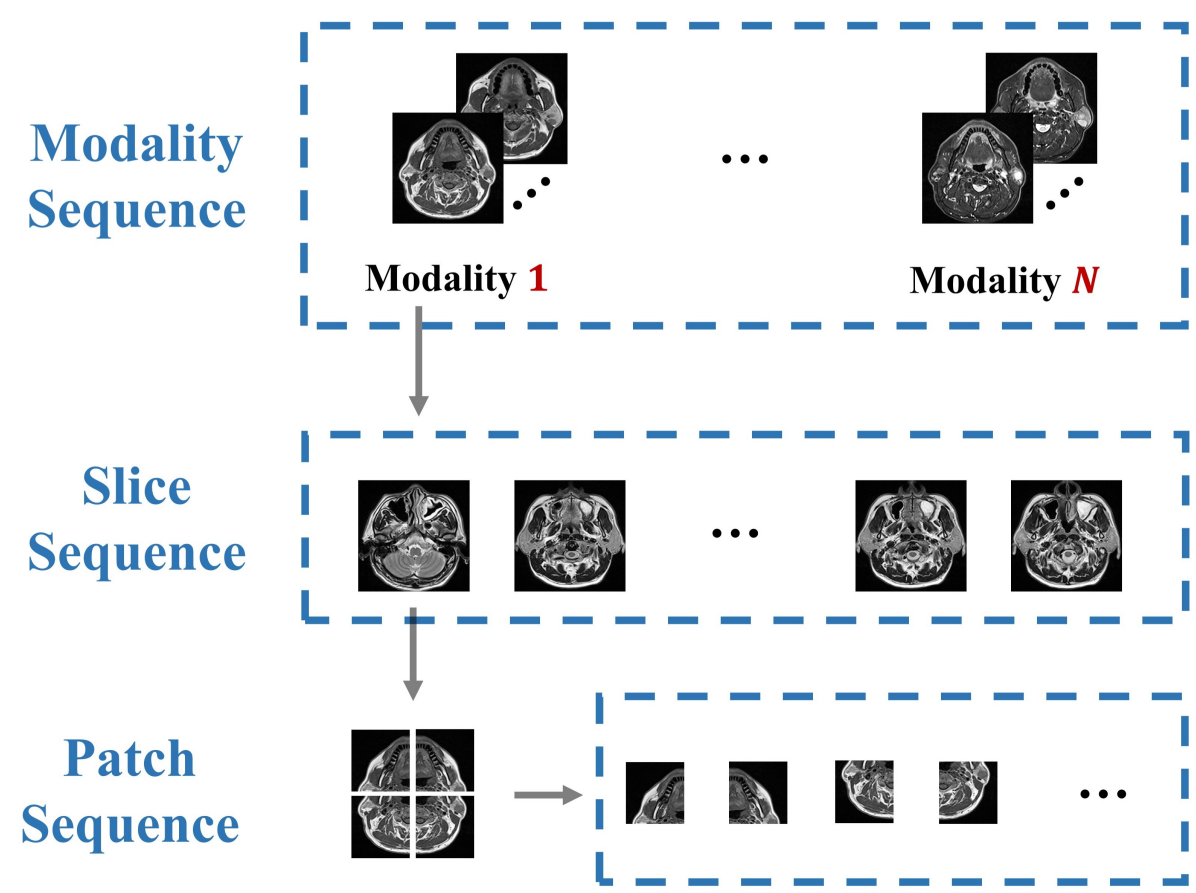

Figure 1. Compared with natural images, multi-modal medical images have more informative sequences.

In summary, we make the following two contributions:

1. We apply transformers to multi-modal medical image classification for the first time and significantly improve the performance of deep models with low computational cost.

2. We propose a novel multi-modal image fusion strategy in this work, which can be leveraged to capture mutual information from images of different modalities in a more efficient way.

The rest of this paper is organized as follows. Section 2 presents some closely related works. The pipeline of our proposed method is in Section 3. Section 4 introduces the experimental results and details. We discuss the results in Section 5. Finally, we summarize our work in Section 6.

\section{Related Work}

\subsection{Multi-Modal Medical Image Analysis}

Multi-modal medical analysis is one of the most fundamental and challenging parts of medical image analysis [8]. It is proved that a reasonable fusion of different modalities has been a potential means to enhance deep networks [6]. Multi-modal fusion can capture more abundant pathological information and improve the quality of diagnosis $[9,10]$.

Some works mainly used the input-level fusion, which is the most common fusion method in multi-modal medical image analysis [11-14]. Some other papers have shown the potential of feature-level fusion in medical image processing. Hyper DenseNet built dual deep networks for different modalities of Magnetic resonance imaging (MRI) and linked features across these streams [15]. Nie et al. [16] fused final features from modality-specific paths to make final decisions. MMFNet used specific encoders to capture modality-specific features and designs a decoder with a complex structure to fuse these features [17]. Different from the first two techniques, $[18,19]$ applied decision-level fusion technology to improve performance. Tseng et al. [19] designed a novel encoder-decoder structure to capture and fuse low-level and high-level features, then the results of each branch were fused to 
generate the final result. Shachor et al. [18] set a gate network to dynamically combine each decision and make a prediction.

Besides, some studies have evaluated multiple fusion methods at the same time. Setio et al. [20] used feature-level fusion and decision-level fusion in their work. Guo et al. [21] designed three kinds of fusion networks, and obtains better performance than a single modality. These fusion methods improve the performance of the model to a certain extent, but there are some shortcomings, such as poor scalability, large computational complexity, and difficulty in establishing long-range connections.

\subsection{Transformers}

Transformers were first proposed for machine translation and achieved satisfactory results in a large number of NLP tasks. For a long time, CNN was considered an irreplaceable basic structure in computer vision tasks [22-25], but currently the breakthrough progress of transformer shows that it is a strong competitor of CNN. Transformers use the self-attention mechanism as the core module to build a convolution-free deep network. Compared with $\mathrm{CNN}$, transformers does not require human-defined inductive bias, and it can handle long-range dependencies well.

Before transformers became popular in the computer vision community, attention and self-attention mechanisms were long used as the auxiliary module of CNN in medical image analysis and greatly improved the performance of deep models. MADGAN [26] integrated the self-attention module into the generative adversarial network for unsupervised medical anomaly detection. Liu et al. [27] developed a CNN with a novel feature pyramid attention mechanism for automatic segmentation of the prostate. Wu et al. [28] proposed a new automated framework that improves the standard U-Net-based architecture through attention modules to accurately delineate epicardial and endocardial contours. Yang et al. [29] presented an advanced segmentation method based on the recursive attention model to segment the left atrium.

The success of attention and self-attention mechanisms motivates researchers to directly apply transformers to computer vision. Some work uses the framework of CNN and transformer [2,30,31], while others directly use pure transformers to replace $\mathrm{CNN}[2,4,5,32]$.

Due to its good performance and superiority in capturing long-range dependencies, Transformers have been widely used in medical image segmentation. TransUNet [33] is the first transformer-based medical image segmentation framework, which uses the transformer to encode the global context. CoTr [34] presented a novel framework that efficiently bridges a CNN and a transformer for 3D medical image segmentation. UNETR [35] utilizes a pure transformer as the encoder to effectively capture the multi-scale information.

These methods have shown encouraging results in computer vision tasks and medical image segmentation, but their direct applications in multi-modal medical images are not effective and require a lot of computing resources. As far as we know, TransMed is the first multi-modal medical image classification framework based on transformers, which provides a novel multi-modal image fusion strategy.

\section{Methods}

The structure of TransMed is shown in Figure 2. The most common method of multimodal medical image classification is to train CNN directly (such as Resnet [36]). Firstly, the image is encoded as a high-level feature representation, and then its features or decisions are fused. Different from the existing methods, our method uses transformers to introduce the self-attention mechanism into the multi-modal fusion strategy. We will first introduce how to directly apply transformers to aggregate feature representations from decomposed image patches in Section 3.1. Then, the overall framework of TransMed will be described in detail in Section 3.2. 


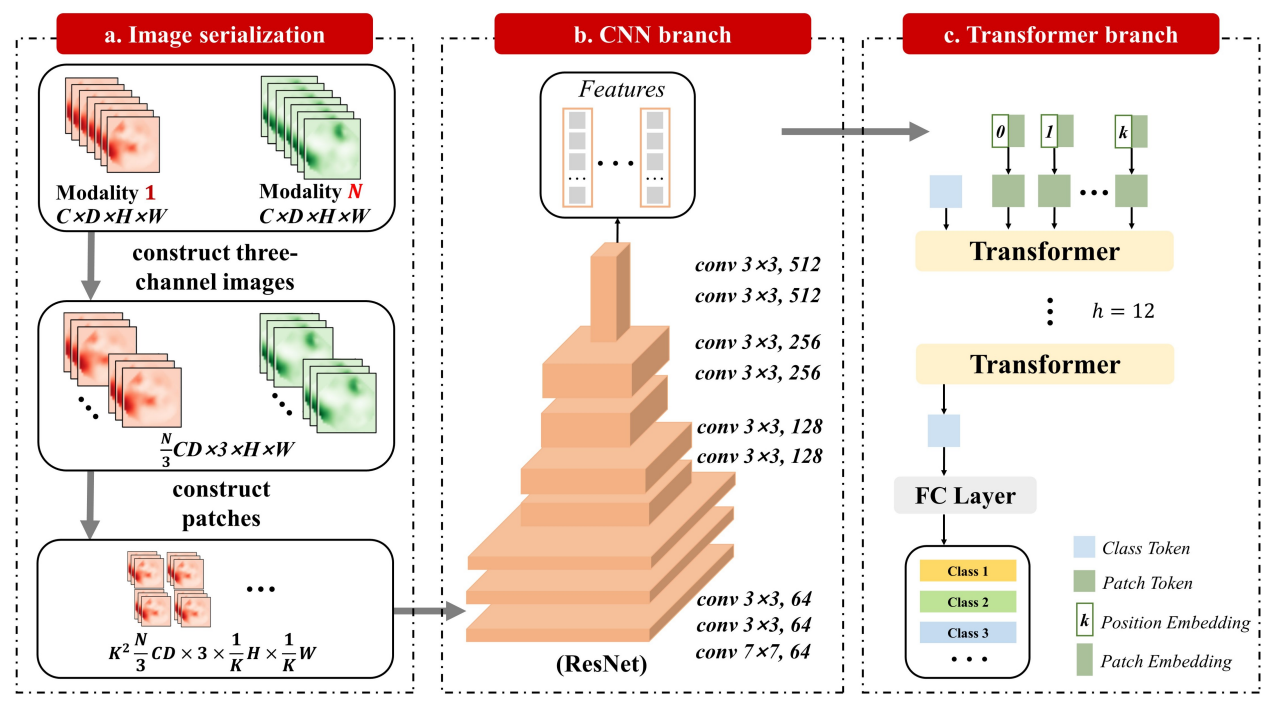

Figure 2. Overview of TransMed, which is composed of CNN branch and transformer branch.

\subsection{Transformers Aggregate Multi-Modal Features}

In this work, we follow the original DeiT implementation as much as possible. The advantage of this intentionally simple setting is to reduce the impact of other tricks on the performance of the model and intuitively show the benefits of transformers. In addition, we can use the extensible DeiT model and its pre-trained weights almost immediately.

The structure of the transformer is shown in Figure 3a. The important components of the transformer including self-attention (SA), multi-head self-attention (MSA), and multilayer perception (MLP). The input of transformers includes a variety of embeddings and tokens. Slightly different from DeiT, we remove the linear projection layer and distillation token. We will describe each of these components in this section.

\subsubsection{Self-Attention}

SA is an attention mechanism, which uses other parts of the same sample to predict the rest of the data sample. In computer vision, it is a little similar to non-local networks [37]. SA has many forms, and the common transformer relies on the form of scaled dot-product shown in Figure $3 b$. In the SA layer, the input vector $X$ is first transformed into three different vectors: query matrix $\mathrm{Q}$, key matrix $\mathrm{K}$, and value matrix $\mathrm{V}$ :

$$
Q=X W_{q}, K=X W_{k}, V=X W_{v}
$$

where $W_{q}, W_{k}$, and $W_{v}$ are trainable matrices. Then, the weight assigned to each value is determined by the dot product of the query and the corresponding key. The attention function between different input vectors is calculated as follows:

$$
\operatorname{Attention}(Q, K, V)=\operatorname{Softmax}\left(\frac{Q K^{\mathrm{T}}}{\sqrt{d_{k}}}\right) \cdot V
$$

where $d_{k}$ is the dimension of key vector $k . \sqrt{d_{k}}$ provides an appropriate normalization to make the gradient more stable. 


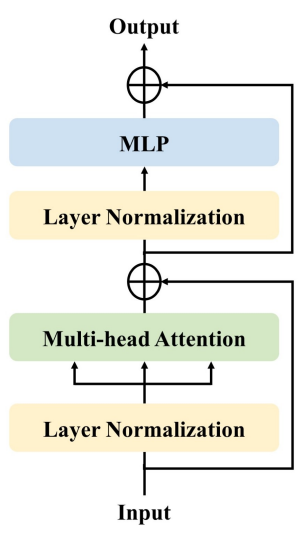

(a)

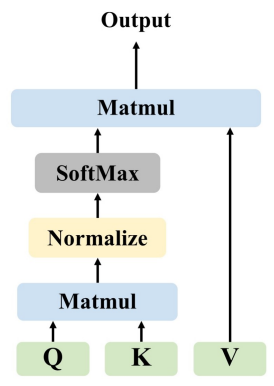

(b)

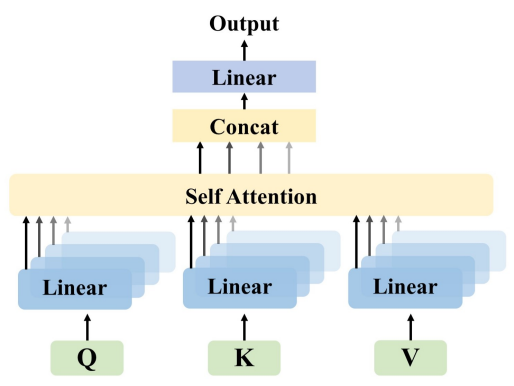

(c)

Figure 3. (a) Structure of the transformer. (b) Overview of self-attention, matmul means matrix product of two arrays. (c) An illustration of our multi-head self-attention component, concat means concatenate representations.

\subsubsection{Multi-Head Self-Attention}

MSA is the core component of the transformer. As shown in Figure 3c, the difference from SA is that the multi-head mechanism splits the input into many small parts, then calculates the scaled dot-product of each input in parallel, and splices all the attention outputs to get the final result. The formula of MSA can be written as follows:

$$
\begin{gathered}
\text { head }_{i}=\operatorname{Attention}\left(Q W_{i}^{Q}, K W_{i}^{K}, V W_{i}^{V}\right) \\
M S A(Q, K, V)=\text { Concat }\left(\text { head }_{1}, \ldots, \text { head }_{i}\right) W^{O}
\end{gathered}
$$

where the projections $W_{i}^{Q}, W_{i}^{K}, W_{i}^{V}$, and $W^{O}$ are trainable parameter matrices; $h$ is the number of transformer layers. The advantage of MSA is that it allows the model to learn sequence and location information in different representation subspaces.

\subsubsection{Multi-Layer Perceptron}

In this paper, an MLP is added on top of the MSA layer. The MLP is composed of linear layers separated by a GeLU [38] activation. Both MSA and MLP have skip-connections like residual networks and with a layer normalization. Therefore, it is assumed that the representation of the $t-1$ layer is $x_{t-1}$, LN represents the linear normalization, and the output of the $t$ layer can be written as follows:

$$
\begin{gathered}
\hat{x}_{t}=\operatorname{MSA}\left(\operatorname{LN}\left(x_{t-1}\right)\right)+x_{t-1} \\
x_{t}=\operatorname{MLP}\left(\operatorname{LN}\left(\hat{x}_{t}\right)\right)+\hat{x}_{t}
\end{gathered}
$$

\subsubsection{Embeddings and Tokens}

The input layer contains five embeddings and tokens, which are patch embedding, position embedding, class embedding, patch token, and class token.

Patch embedding is the representation of each patch's output from CNN, and class embedding is a trainable vector. To encode the spatial information and location information of a patch into patch tokens, we use position embeddings and patch embeddings to preserve the information. Class embedding does not have patch embedding that can be added, so class token and class embedding are equivalent. Suppose the input is $x$, the trainable vector is $W^{c}$, the position embedding is $x_{p o}$, patch tokens $x_{p t}$ and class token $x_{c t}$ can be expressed as follows:

$$
\begin{gathered}
x_{p t}=\operatorname{Conv}(x)+x_{p o} \\
x_{c t}=W^{c}
\end{gathered}
$$


The class token is attached to patch tokens before the input layer of transformers, passes through the transformer layer, and then outputs from the fully connected layer to predict the class.

\subsection{TransMed}

Instead of using pure transformers as the encoder, TransMed adopts a hybrid model including CNN and transformer, in which CNN is used as a low-level feature extractor [39] to generate the patch embedding.

Given a multi-modal image $x \in R^{N \times C \times D \times H \times W}$, where spatial resolution is $H \times W$, the depth is $D$, the number of channels is $C$, and the number of modalities is $N$. Before sending it to the CNN encoder, it is necessary to construct the sequence. First, combine the channel dimension, depth dimension, and modality dimension of a multi-modal image to obtain $x^{\prime} \in R^{(N \times C \times D) \times H \times W}$. Then, three adjacent 2D slices of a multi-modal image are superimposed to construct three-channel images $x^{\prime \prime} \in R^{(1 / 3 \times N \times C \times D) \times 3 \times H \times W}$. Then, according to [4], each image will be divided into $K \times K$. The larger $K$ value means that the size of each patch is smaller. We will evaluate the impact of different $K$ values on the performance of the model in Section 4. Finally, the image is encoded into a patch $x_{\text {input }} \in R^{\left(1 / 3 \times N \times C \times D \times K^{2}\right) \times 3 \times(H / K) \times(W / K)}$.

After the image sequence is constructed, it is input into the 2D CNN. The last fully connected layer of $2 \mathrm{D} \mathrm{CNN}$ is replaced by a linear projection layer to map the features of the vector patch to the potential embedding space. The 2D CNN extracts low-level features from the image sequence and encodes them preliminarily.

\section{Results}

To evaluate the proposed method, we carry out comprehensive experiments on the parotid gland tumor (PGT) dataset and the MRNet dataset. Experimental results demonstrate that TransMed achieves state-of-the-art performance on two datasets. In the following, we first introduce the datasets and preprocessing details. Next, we introduce the experimental settings and evaluation criteria. Then we present a comparison of our model with some state-of-the-arts on the two datasets. Finally, we perform a series of ablation experiments on the PGT dataset.

\subsection{Dataset}

\subsubsection{PGT Dataset}

The PGT dataset contains 344 head and neck MRI examinations carried out at the Stomatological Hospital of China Medical University. The ethics board approved the use of the images for this research. This dataset includes two modalities of MRI (T1 and T2), as shown in Figure 4. The ground truth labels are obtained from biopsies.

The incidence of malignant tumors in PGT is about 20\% [40]. Correct preoperative diagnosis of these tumors is essential for proper surgical planning. Among them, imaging examination plays an important role in determining the nature of parotid gland masses. MRI is considered to be the preferred imaging method for preoperative diagnosis of PGT [41]. It has been proved in previous studies that in radiation therapy, MRI-based tissue characterization and segmentation are of vital importance for improving the treatment $[42,43]$. MRI can provide information about the exact location of the lesion, the relationship with the surrounding structure and can assess the spread of nerves and bone invasion. However, it is reported that the PGT shows considerable overlap in imaging features (such as tumor margins, homogeneity, and signal intensity), so it is difficult for doctors to identify the mass.

According to common clinical classifications, we divide PGT into five categories: Pleomorphic Adenoma (PA), Warthin Tumor (WT), Malignant Tumor (MT), Basal Cell Adenoma (BCA), and Other Benign Lesions (OBL) [44]. In the PGT dataset, the patients are randomly divided into the training set $(n=241)$, validation set $(n=34)$, and an independent test set $(n=69)$ according to the ratio of 7:1:2. In this study, we use stratified random 
sampling to ensure that at least 5 and 10 positive examples of each label are present in the validation and test set, respectively. The training set is used to optimize the model parameters, and the validation set is used to select the best model.

In the data preprocessing stage, we first perform OTSU [45] to extract the foreground area in the original image. Then the images of different modalities of the same patient are registered to improve the consistency of the foreground area. Then resample each image to $18 \times 448 \times 448$. Therefore, each image is a stack of 3D images of MRI T1 and T2, and the size is $36 \times 448 \times 448$. Data augmentation uses random flipping and random noise. Random flipping performs flipping of the image with $50 \%$ probability. Random noise adds Gaussian noise with a mean value of 0 and a variance of 0.1 to the image.

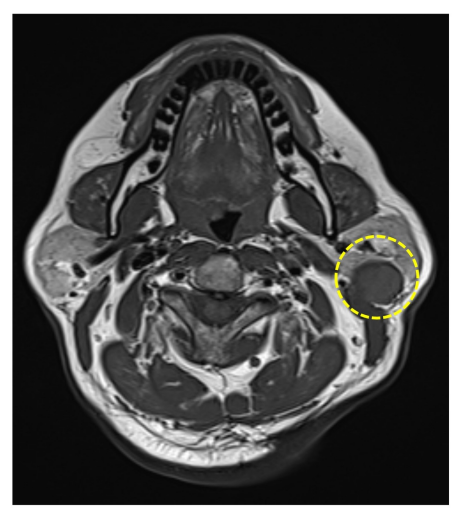

Modality 1: T1 MRI

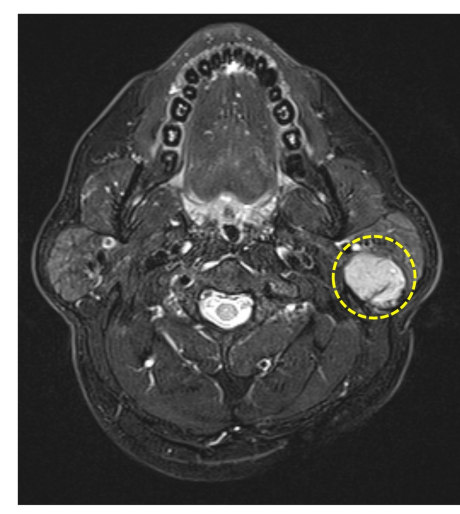

Modality 2: T2 MRI

Figure 4. An illustration of the images in the PGT dataset. The yellow circle represents the location of the tumor.

\subsubsection{MRNet Dataset}

The MRNet Dataset contains 1370 knee MRI examinations that were carried out at the Stanford University Medical Center [46]. Each case was labeled according to the anterior cruciate ligament (ACL) tear, meniscus tear, or other signs of abnormalities in the corresponding knee (abnormal). The author randomly split the dataset into 1130 training cases, 120 validation cases, and 120 test cases. The provided dataset includes three modalities of MRI (T1-weighted images, T2-weighted images, and proton density-weighted). Each image is of size $256 \times 256$ and the number of slices ranges between 17 and 61 . The data were preprocessed by applying same procedures used in the MRNet. Data augmentation strategy is consistent with the strategy used in the PGT dataset.

\subsection{Experimental Settings and Evaluation Criteria}

We set SGD as the optimizer with a momentum equal to 0.7. The learning rate is $10^{-3}$, and the maximum training round is 100 . The patch size is set to 2 . Our experiments were carried out on NVIDIA 3080 GPU. The code is implemented using PyTorch [47] and TorchIO [48]. To eliminate accidental factors, each model is subjected to 10 independent experiments, and other experimental parameters keep consistent during training.

To evaluate the performance of the model, we select the Accuracy (ACC) and Precision (PR) as the evaluation criteria in the PGT dataset. In the MRNet dataset, to produce comparable results with other baseline methods, we use ACC, Area Under Curve (ROCAUC, or AUC), Sensitivity (SE), and Specificity (SP) as evaluation criteria. TP, TN, FP, and FN are the number of true positive, true negative, false positive, and false negative. ACC is defined as the ratio of the number of correctly classified samples to the total number of samples.

$$
A C C=\frac{T P+T F}{T P+T N+F P+F N}
$$


SE is defined as calculating the ratio that is correctly classified as positive to those that are true positive samples.

$$
S E=\frac{T P}{T P+F N}
$$

$\mathrm{SP}$ is defined as calculating the ratio that is correctly classified as negative to those that are true negative samples.

$$
S P=\frac{T N}{T N+T P}
$$

$\mathrm{PR}$ is defined as calculating the ratio of samples correctly classified as positive to all samples predicted to be positive.

$$
P R=\frac{T P}{T P+F P}
$$

AUC is used to evaluate the quality of the binary classification model, which is defined as the area under the ROC curve.

\subsection{Baseline Methods}

\subsubsection{PGT Dataset}

The input-level fusion strategy and the decision-level fusion strategy can be implemented using mainstream 2D CNN and 3D CNN, so the selected network includes Resnet34, Resnet152, 3D Resnet34, P3D [49], C3D [50], and BoTNet50 [31]. In feature-level fusion experiments, we used two common feature-level fusion methods $[15,16]$. Since these two papers focus on segmentation tasks, we modify the network structure to adapt to the classification tasks.

\subsubsection{MRNet Dataset}

In the experiments, we compare our method with three state-of-the-art models: MRNet [46], ELNet [51], and MRPyrNet [52]. MRNet mainly includes three AlexNets [53], which independently make predictions for each modality and use decision-level fusion strategy. ELNet changes the backbone network from AlexNet to Resnet and proposed two technologies: multi-slice normalization and BlurPool layers to improve performance. MRPyrNet uses a Feature Pyramid Network and Pyramidal Detail Pooling to gather and capture small appearing injuries in the knee area. The model was inserted into MRNet and ELNet and achieved significant performance improvement.

\subsection{Experimental Results \\ 4.4.1. PGT Dataset}

Table 1 reports the performance of our proposed models, in which three variants are provided: the tiny version (TransMed-T) use ResNet18 and DeiT-Tiny (DeiT-T) as backbones for CNN branch and transformer branch, respectively; the small version (TransMed-S) use ResNet34 and DeiT-Small (DeiT-S) as backbone; the base version (TransMed-B) uses ResNet50 and DeiT-Base (DeiT-B) as the backbone.

TransMed consistently outperforms previous multi-modal fusion strategies by a large margin. The confusion matrix of TransMed-S is shown in Figure 5, it achieves on average about $10.1 \%$ improvement in terms of the average accuracy with respect to the BoTNet, while the larger version TransMed-B slightly suffers from overfitting on the dataset. Table 1 also compares the number of parameters and computational costs between our proposed models and previous methods. TransMed achieves state-of-the-art performance with much fewer parameters and computational costs. TransMed is highly efficient as it models the long-range relationship between modalities very well. 
Table 1. Comparison on the PGT dataset (average ACC \% and PR \% for each disease. IF, FF, and DF represent input-level fusion, feature-level fusion and decision-level fusion, respectively). All the results are presented as mean \pm standard deviation. The throughput is calculated as the time consumption (second) of predicting 100 images, which is measured using 2080Ti GPU.

\begin{tabular}{|c|c|c|c|c|c|c|c|c|c|c|}
\hline Method & Dim & Fusion & Params & Throughput & ACC & PA & WT & MT & BCA & OBL \\
\hline P3D & $3 \mathrm{D}$ & IF & $67 \mathrm{M}$ & 15.8 & $76.1 \pm 5.5$ & $59.9 \pm 23.1$ & $84.3 \pm 5.3$ & $69.7 \pm 19.0$ & $71.4 \pm 7.3$ & $78.0 \pm 14.0$ \\
\hline $\mathrm{C} 3 \mathrm{D}$ & $3 \mathrm{D}$ & IF & $28 \mathrm{M}$ & 32.3 & $71.0 \pm 4.1$ & $68.3 \pm 38.9$ & $81.3 \pm 15.4$ & $67.8 \pm 10.0$ & $71.4 \pm 7.5$ & $84.5 \pm 12.4$ \\
\hline Resnet34 & $2 \mathrm{D}$ & IF & $22 \mathrm{M}$ & 4.0 & $69.9 \pm 4.0$ & $81.0 \pm 11.0$ & $77.6 \pm 7.4$ & $61.4 \pm 10.8$ & $53.8 \pm 15.5$ & $68.1 \pm 7.9$ \\
\hline Resnet152 & $2 \mathrm{D}$ & IF & $58 \mathrm{M}$ & 6.3 & $69.0 \pm 3.5$ & $50.5 \pm 18.0$ & $74.1 \pm 10.1$ & $64.3 \pm 20.0$ & $62.9 \pm 8.9$ & $75.2 \pm 11.4$ \\
\hline 3D Resnet34 & $3 \mathrm{D}$ & IF & $64 \mathrm{M}$ & 23.8 & $73.3 \pm 5.1$ & $69.2 \pm 16.2$ & $86.8 \pm 5.2$ & $75.3 \pm 18.1$ & $68.7 \pm 13.8$ & $65.7 \pm 6.4$ \\
\hline BoTNet50 & $3 \mathrm{D}$ & IF & $21 \mathrm{M}$ & 4.2 & $77.4 \pm 2.0$ & $82.6 \pm 14.4$ & $84.0 \pm 4.1$ & $76.1 \pm 6.9$ & $76.0 \pm 12.4$ & $70.8 \pm 8.4$ \\
\hline [15] & $3 \mathrm{D}$ & $\mathrm{FF}$ & $45 \mathrm{M}$ & 27.9 & $74.2 \pm 2.9$ & $76.0 \pm 24.8$ & $86.2 \pm 10.0$ & $72.9 \pm 16.3$ & $75.2 \pm 24.6$ & $80.0 \pm 15.2$ \\
\hline [16] & $3 \mathrm{D}$ & $\mathrm{FF}$ & $130 \mathrm{M}$ & 30.8 & $73.3 \pm 2.4$ & $46.2 \pm 13.2$ & $78.4 \pm 7.9$ & $70.2 \pm 15.5$ & $69.8 \pm 15.8$ & $79.0 \pm 10.7$ \\
\hline P3D & $3 \mathrm{D}$ & DF & $136 \mathrm{M}$ & 22.1 & $74.8 \pm 4.6$ & $50.5 \pm 20.0$ & $85.1 \pm 4.4$ & $70.5 \pm 20.2$ & $69.5 \pm 8.7$ & $73.4 \pm 14.3$ \\
\hline C3D & $3 \mathrm{D}$ & DF & $57 \mathrm{M}$ & 41.3 & $71.0 \pm 3.3$ & $58.3 \pm 33.3$ & $70.7 \pm 9.3$ & $74.0 \pm 8.5$ & $78.9 \pm 20.0$ & $73.2 \pm 6.7$ \\
\hline Resnet34 & $2 \mathrm{D}$ & DF & $45 \mathrm{M}$ & 5.6 & $71.3 \pm 4.5$ & $72.7 \pm 21.7$ & $75.3 \pm 8.1$ & $72.5 \pm 10.3$ & $60.9 \pm 16.8$ & $70.3 \pm 9.7$ \\
\hline Resnet152 & $2 \mathrm{D}$ & DF & $116 \mathrm{M}$ & 9.5 & $72.2 \pm 5.5$ & $63.5 \pm 18.3$ & $75.6 \pm 10.4$ & $73.4 \pm 18.7$ & $83.2 \pm 16.3$ & $69.6 \pm 11.5$ \\
\hline 3D Resnet34 & $3 \mathrm{D}$ & DF & $128 \mathrm{M}$ & 34.6 & $72.1 \pm 3.5$ & $64.7 \pm 14.4$ & $81.5 \pm 9.3$ & $66.8 \pm 8.4$ & $69.6 \pm 8.9$ & $72.1 \pm 14.9$ \\
\hline BoTNet50 & $3 \mathrm{D}$ & DF & $44 \mathrm{M}$ & 6.6 & $78.8 \pm 3.4$ & $72.6 \pm 6.2$ & $82.9 \pm 4.3$ & $73.2 \pm 8.2$ & $76.9 \pm 16.2$ & $87.9 \pm 8.2$ \\
\hline TransMed-T & $2 \mathrm{D}$ & 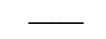 & $17 \mathrm{M}$ & 4.2 & $87.0 \pm 2.6$ & $80.1 \pm 13.8$ & $87.3 \pm 3.0$ & $90.7 \pm 5.1$ & $82.5 \pm 15.3$ & $93.6 \pm 3.3$ \\
\hline TransMed-S & $2 \mathrm{D}$ & - & $43 \mathrm{M}$ & 4.5 & $88.9 \pm 3.0$ & $90.1 \pm 12.2$ & $89.2 \pm 6.8$ & $92.0 \pm 4.4$ & $82.9 \pm 9.3$ & $88.3 \pm 6.1$ \\
\hline TransMed-B & $2 \mathrm{D}$ & $\longrightarrow$ & $110 \mathrm{M}$ & 6.3 & $87.4 \pm 2.1$ & $86.2 \pm 15.2$ & $88.4 \pm 3.8$ & $88.2 \pm 7.0$ & $84.8 \pm 13.8$ & $92.2 \pm 8.0$ \\
\hline
\end{tabular}

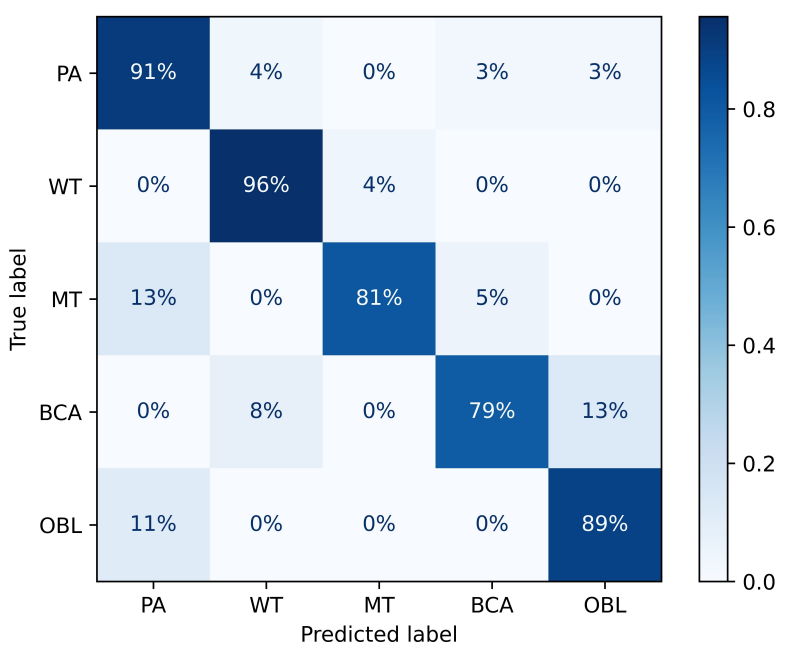

Figure 5. Confusion matrix of TransMed-S on the PGT dataset.

\subsubsection{MRNet Dataset}

Table 2 reports the performance of MRNet, ELNet, MRPyrNet, and TransMed in the MRNet dataset. Compared with the best performance of the three baseline methods, TransMed achieved 3.5\%, 0.5\%, and 5.7\% improvements in abnormality, ACL tear, and meniscus tear, respectively. Our proposed strategy dramatically improves the abnormality and meniscus tear detection of the baseline method, which shows that the transformer structure can improve the diagnostic ability of the model. More importantly, the proposed method is more robust on the SE, representing a better situation for the potential clinical applications of TransMed. Specifically, compared with the previous techniques, TransMed has achieved a performance improvement of 13.6\% (MRPyrNet) to 31.5\% (ELNet) in the sensitivity of ACL tear detection.

It is noteworthy that in the MRNet dataset, our method surpasses ELNet [51] and MRPyrNet [52] without inputting any domain knowledge. In ELNet, an experienced radiologist is asked to determine the most informative slice. MRPyrNet strongly assumes that the anomaly is always in the center of the MRI slice. 


\subsection{Ablation Experiments}

To demonstrate the effect of transformers in TransMed, we perform ablation experiments on PGT dataset. For TransMed, changing the backbone from TransMed-T to TransMed-S results in 1.9\% improvement in average accuracy, at the expense of a much larger computational cost. Therefore, considering the computation cost, all experimental comparisons in this paper are conducted with TransMed-T to demonstrate the effectiveness of TransMed.

In the experiment, TransMed's CNN and transformers were removed, respectively, and all other conditions remained unchanged. The results are shown in Table 3. The results indicate that the transformer greatly improves the ability of the deep model to explore the relationship between modalities with little increase of parameters and computation. However, the performance of the pure transformer structure is poor due to the small dataset.

We also explored the impact of different patch sizes on performance in image serialization by changing $K$ values, respectively, while other conditions remain unchanged. The results are shown in Table 4. The experimental results show that the performance is poor when the $K$ value is large. The possible reason is that too small image patches destroy the semantic information of the image.

Table 2. Comparison on the MRNet dataset.

\begin{tabular}{|c|c|c|c|c|c|}
\hline Pathology & Method & ROC-AUC & ACC & SE & SP \\
\hline \multirow{7}{*}{ Abnormality } & MRNet & 0.936 & 0.883 & 0.947 & 0.64 \\
\hline & ELNet & 0.941 & 0.917 & 0.968 & 0.72 \\
\hline & MRPyrNet (with MRNet) & & & $\longrightarrow$ & - \\
\hline & MRPyrNet (with ELNet) & & & & \\
\hline & TransMed-T (Ours) & $0.974 \pm 0.007$ & $0.907 \pm 0.010$ & $0.955 \pm 0.002$ & $0.728 \pm 0.016$ \\
\hline & TransMed-S (Ours) & $0.976 \pm 0.004$ & $0.918 \pm 0.006$ & $0.958 \pm 0.011$ & $0.720 \pm 0.000$ \\
\hline & TransMed-B (Ours) & $0.958 \pm 0.018$ & $0.898 \pm 0.012$ & $0.951 \pm 0.016$ & $0.696 \pm 0.020$ \\
\hline \multirow{7}{*}{ ACL Tear } & MRNet & $0.955 \pm 0.005$ & $0.847 \pm 0.005$ & $0.722 \pm 0.000$ & $0.950 \pm 0.009$ \\
\hline & ELNet & $0.940 \pm 0.001$ & $0.808 \pm 0.000$ & $0.648 \pm 0.019$ & $0.939 \pm 0.015$ \\
\hline & MRPyrNet (with MRNet) & $0.976 \pm 0.003$ & $0.886 \pm 0.010$ & $0.815 \pm 0.019$ & $0.944 \pm 0.009$ \\
\hline & MRPyrNet (with ELNet) & $0.960 \pm 0.015$ & $0.881 \pm 0.034$ & $0.827 \pm 0.039$ & $0.924 \pm 0.030$ \\
\hline & TransMed-T (Ours) & $0.969 \pm 0.009$ & $0.938 \pm 0.009$ & $0.935 \pm 0.021$ & $0.939 \pm 0.008$ \\
\hline & TransMed-S (Ours) & $0.981 \pm 0.011$ & $0.949 \pm 0.003$ & $0.963 \pm 0.007$ & $0.938 \pm 0.005$ \\
\hline & TransMed-B (Ours) & $0.949 \pm 0.013$ & $0.931 \pm 0.012$ & $0.924 \pm 0.027$ & $0.936 \pm 0.006$ \\
\hline \multirow{7}{*}{ Meniscus Tear } & MRNet & $0.843 \pm 0.016$ & $0.778 \pm 0.027$ & $0.750 \pm 0.067$ & $0.799 \pm 0.009$ \\
\hline & ELNet & $0.869 \pm 0.031$ & $0.775 \pm 0.044$ & $0.814 \pm 0.109$ & $0.745 \pm 0.075$ \\
\hline & MRPyrNet (with MRNet) & $0.889 \pm 0.006$ & $0.808 \pm 0.008$ & $0.853 \pm 0.048$ & $0.775 \pm 0.052$ \\
\hline & MRPyrNet (with ELNet) & $0.895 \pm 0.008$ & $0.761 \pm 0.042$ & $0.872 \pm 0.106$ & $0.676 \pm 0.149$ \\
\hline & TransMed-T (Ours) & $0.939 \pm 0.015$ & $0.830 \pm 0.024$ & $0.869 \pm 0.018$ & $0.800 \pm 0.032$ \\
\hline & TransMed-S (Ours) & $0.945 \pm 0.011$ & $0.848 \pm 0.016$ & $0.881 \pm 0.037$ & $0.824 \pm 0.026$ \\
\hline & TransMed-B (Ours) & $0.952 \pm 0.012$ & $0.853 \pm 0.018$ & $0.879 \pm 0.039$ & $0.834 \pm 0.007$ \\
\hline
\end{tabular}

Table 3. Ablation study on the effectiveness of CNN branch and transformer branch.

\begin{tabular}{ccccccccc}
\hline Model & Params & TFlops & Acc & PA & WT & MT & BCA & OBL \\
\hline TransMed-T & $17 \mathrm{M}$ & 0.09 & $87.0 \pm 2.6$ & $80.1 \pm 13.8$ & $87.3 \pm 3.0$ & $90.7 \pm 5.1$ & $82.5 \pm 15.3$ & $93.6 \pm 3.3$ \\
w/o transformer & $12 \mathrm{M}$ & 0.01 & $71.3 \pm 2.5$ & $71.6 \pm 17.8$ & $78.9 \pm 3.9$ & $73.9 \pm 12.5$ & $66.9 \pm 13.1$ & $69.9 \pm 13.3$ \\
w/o CNN & $5 \mathrm{M}$ & 0.07 & $51.3 \pm 5.9$ & $20.0 \pm 18.7$ & $61.5 \pm 13.5$ & $37.0 \pm 5.7$ & $41.7 \pm 40.1$ & $51.0 \pm 16.4$ \\
\hline
\end{tabular}

Table 4. Ablation study on different patch sizes.

\begin{tabular}{cccccccc}
\hline Model & K & ACC & PA & WT & MT & BCA & OBL \\
\hline TransMed-T & 1 & $86.8 \pm 2.3$ & $83.1 \pm 12.4$ & $90.1 \pm 3.9$ & $88.7 \pm 10.1$ & $78.3 \pm 13.7$ & $95.3 \pm 5.9$ \\
TransMed-T & 2 & $87.0 \pm 2.6$ & $80.1 \pm 13.8$ & $87.3 \pm 3.0$ & $90.7 \pm 5.1$ & $82.5 \pm 15.3$ & $93.6 \pm 3.3$ \\
TransMed-T & 4 & $86.4 \pm 3.3$ & $86.3 \pm 17.2$ & $87.0 \pm 6.5$ & $89.5 \pm 3.6$ & $75.5 \pm 8.3$ & $92.1 \pm 7.2$ \\
TransMed-T & 8 & $80.0 \pm 5.8$ & $81.2 \pm 17.4$ & $81.1 \pm 5.3$ & $88.5 \pm 4.2$ & $63.9 \pm 12.1$ & $88.2 \pm 13.6$ \\
TransMed-T & 16 & $65.2 \pm 5.8$ & $49.0 \pm 32.2$ & $72.1 \pm 7.7$ & $70.4 \pm 7.9$ & $62.3 \pm 23.0$ & $64.1 \pm 14.5$ \\
\hline
\end{tabular}




\section{Discussion}

Mining different sequences of information in multi-modal medical images are essential to improve the performance of deep models. Our work applies transformers to multi-modal medical image classification for the first time because it can effectively explore sequence information that is difficult to capture by CNN. In this work, we first use ResNet to extract the image features and then use Transformer to capture the long-range dependency between the sequences. The experimental results show that our method surpasses the previous state-of-the-art model in the two datasets and has high stability. This study indicates that a hybrid architecture based on $\mathrm{CNN}$ and transformers can significantly improve multi-modal medical image classification performance.

To verify the validity of the model, we implement two ablation experiments. The first ablation experiment shows that there is still a big gap between the pure transformer and the typical CNN in small medical image datasets. The reason for the poor performance of the standard vision transformer structure is that the self-attention mechanism does not have an inductive bias similar to the $\mathrm{CNN}$ structure. Although when the amount of data is large enough, the transformer structure is proven to surpass the domain knowledge brought by the inductive bias [4]. However, the medical image dataset is small and cannot achieve satisfactory performance.

The second ablation experiment shows that the gain of serializing two-dimensional images is marginal ( $87.0 \%$ vs. $86.8 \%)$. Moreover, the model's performance tends to decline with the increase of $K$. In our work, we follow the existing technology of image serialization in the natural image classification model based on transformers. However, in medical images, such techniques may lead to the degradation of model performance. Because image serialization will separate the tumor, thus the separated tumor area is difficult to recognize as a tumor.

In summary, the preliminary results of our proposed method are encouraging, but there are still many challenges. In order to make the transformer-based structure better used in medical image analysis, it is very important to modify it according to the specific task.

In future work, we will try to overcome the limitations of current research. The first is to apply TransMed to more medical image datasets of different modalities (such as Computed Tomography (CT) and Positron Emission Computed Tomography (PET)) to further investigate the superiority of the proposed model. Moreover, It is necessary to build a robust cross-modality, transformer-based model. Second, we should apply TransMed to other medical image analysis tasks, such as tumor segmentation and lesion detection. Third, we will improve the image serialization technology to further adapt to multi-modal medical images. The fourth is to explore the high-performance structure based on pure transformers. Last but not least, as a fast-developing deep network, the transformer structure requires a reliable and mature visualization technology to improve the interpretability of the model and intuitively illustrate its advantages in capturing long-range dependencies of multi-modal images. Therefore, our future work includes proposing or improving visualization techniques suitable for transformer-based medical image analysis.

\section{Conclusions}

The transformer is a powerful deep neural network structure for processing sequences in NLP, but it has received little attention in medical image analysis. In this paper, we propose TransMed, which is a novel design of multi-modal medical image classification based on transformers. Unlike CNN-based methods, TransMed uses a hybrid model including $\mathrm{CNN}$ and transformer. Among them, $\mathrm{CNN}$ is used as a low-level feature extractor to generate local feature sequences of multi-modal images; while transformers effectively extract long-range dependencies between sequences from low-level feature sequences to achieve good performance. In the two multi-modal medical image classification datasets, our method achieved an average accuracy improvement of $10.1 \%$ and $1.9 \%$, respectively, compared with the previous state-of-the-art models. Our experiments provided insights on 
the inclusion of transformers in deep networks for medical image analysis, particularly in multi-modal scenarios. Combining these promising results, we believe that the transformer structure has tremendous potential in a large number of medical image analysis tasks.

Author Contributions: Methodology, Y.G. and Y.D.; formal analysis, Y.G.; investigation, Y.D.; resources, F.L.; data curation, F.L.; writing-original draft preparation, Y.G.; writing-review and editing, Y.D. and F.L.; supervision, Y.D. and F.L.; funding acquisition, Y.D. All authors have read and agreed to the published version of the manuscript.

Funding: This research was funded in part by the Youth Program of National Natural Science Foundation of China grant number 61902058, in part by the Fundamental Research Funds for the Central Universities grant number N2019002, in part by the Natural Science Foundation of Liaoning Province grant number No. 2019-ZD-0751, in part by the Fundamental Research Funds for the Central Universities grant number No. JC2019025, in part by the Medical Imaging Intelligence Research grant number N2124006-3, in part by the National Natural Science Foundation of China grant number 61872075 .

Institutional Review Board Statement: The study was conducted according to the guidelines of the Declaration of Helsinki, and approved by the Institutional Review Board of the Stomatological Hospital of China Medical University (March 2021).

Informed Consent Statement: Informed consent was obtained from all subjects involved in the study.

Data Availability Statement: The data presented in this study are available on request from the corresponding author.

Conflicts of Interest: The authors declare no conflict of interest.

\section{References}

1. Vaswani, A.; Shazeer, N.; Parmar, N.; Uszkoreit, J.; Jones, L.; Gomez, A.N.; Kaiser, Ł.; Polosukhin, I. Attention is All you Need. In Proceedings of the Neural Information Processing Systems (NeurIPS), Long Beach, CA, USA, 4-9 December 2017; pp. 5998-6008.

2. Carion, N.; Massa, F.; Synnaeve, G.; Usunier, N.; Kirillov, A.; Zagoruyko, S. End-to-end object detection with transformers. In Proceedings of the European Conference on Computer Vision (ECCV), Glasgow, UK, 23-28 August 2020; pp. $213-229$.

3. Zheng, S.; Lu, J.; Zhao, H.; Zhu, X.; Luo, Z.; Wang, Y.; Fu, Y.; Feng, J.; Xiang, T.; Torr, P.H.S.; et al. Rethinking Semantic Segmentation from a Sequence-to-Sequence Perspective with Transformers. arXiv 2020, arXiv:2012.15840.

4. Dosovitskiy, A.; Beyer, L.; Kolesnikov, A.; Weissenborn, D.; Zhai, X.; Unterthiner, T.; Dehghani, M.; Minderer, M.; Heigold, G.; Gelly, S.; et al. An Image is Worth 16x16 Words: Transformers for Image Recognition at Scale. arXiv 2020, arXiv:2010.11929.

5. Touvron, H.; Cord, M.; Douze, M.; Massa, F.; Sablayrolles, A.; Jegou, H. Training Data-Efficient Image Transformers \& Distillation through Attention. arXiv 2020, arXiv:2012.12877.

6. Zhou, T.; Ruan, S.; Canu, S. A review: Deep learning for medical image segmentation using multi-modality fusion. Array 2019, 3, 100004. [CrossRef]

7. Deng, J.; Dong, W.; Socher, R.; Li, L.J.; Li, K.; Fei-Fei, L. ImageNet: A large-scale hierarchical image database. In Proceedings of the 2009 IEEE Conference on Computer Vision and Pattern Recognition (CVPR), Miami, FL, USA, 20-25 June 2009; pp. 248-255. [CrossRef]

8. Ibtehaz, N.; Rahman, M.S. MultiResUNet: Rethinking the U-Net architecture for multimodal biomedical image segmentation. Neural Netw. 2020, 121, 74-87. [CrossRef] [PubMed]

9. Wang, K.; Zheng, M.; Wei, H.; Qi, G.; Li, Y. Multi-modality medical image fusion using convolutional neural network and contrast pyramid. Sensors 2020, 20, 2169. [CrossRef] [PubMed]

10. Zhu, Z.; Zheng, M.; Qi, G.; Wang, D.; Xiang, Y. A phase congruency and local Laplacian energy based multi-modality medical image fusion method in NSCT domain. IEEE Access 2019, 7, 20811-20824. [CrossRef]

11. Myronenko, A. 3D MRI Brain Tumor Segmentation Using Autoencoder Regularization. In International MICCAI Brainlesion Workshop; Springer: Cham, Switzerland, 2019; Volume 11384, pp. 311-320. [CrossRef]

12. Kamnitsas, K.; Ledig, C.; Newcombe, V.F.J.; Simpson, J.P.; Kane, A.D.; Menon, D.K.; Rueckert, D.; Glocker, B. Efficient multi-scale 3D CNN with fully connected CRF for accurate brain lesion segmentation. Med. Image Anal. 2017, 36, 61-78. [CrossRef]

13. Isensee, F.; Kickingereder, P.; Wick, W.; Bendszus, M.; Maier-Hein, K.H. Brain Tumor Segmentation and Radiomics Survival Prediction: Contribution to the BRATS 2017 Challenge. In Proceedings of the International Conference on Medical Image Computing and Computer Assisted Intervention Workshop (MICCAI), Granada, Spain, 16-20 September 2018 ; pp. $287-297$. [CrossRef]

14. Li, M.; Wang, C.; Zhang, H.; Yang, G. MV-RAN: Multiview recurrent aggregation network for echocardiographic sequences segmentation and full cardiac cycle analysis. Comput. Biol. Med. 2020, 120, 103728. [CrossRef] 
15. Dolz, J.; Gopinath, K.; Yuan, J.; Lombaert, H.; Desrosiers, C.; Ayed, I.B. HyperDense-Net: A Hyper-Densely Connected CNN for Multi-Modal Image Segmentation. IEEE Trans. Med. Imaging 2019, 38, 1116-1126. [CrossRef]

16. Nie, D.; Wang, L.; Gao, Y.; Shen, D. Fully convolutional networks for multi-modality isointense infant brain image segmentation. In Proceedings of the 2016 IEEE 13th International Symposium on Biomedical Imaging (ISBI), Prague, Czech Republic, 13-16 April 2016; pp. 1342-1345. [CrossRef]

17. Chen, H.; Qi, Y.; Yin, Y.; Li, T.; Liu, X.; Li, X.; Gong, G.; Wang, L. MMFNet: A multi-modality MRI fusion network for segmentation of nasopharyngeal carcinoma. Neurocomputing 2020, 394, 27-40. [CrossRef]

18. Shachor, Y.; Greenspan, H.; Goldberger, J. A mixture of views network with applications to multi-view medical imaging. Neurocomputing 2020, 374, 1-9. [CrossRef]

19. Tseng, K.-L.; Lin, Y.-L.; Hsu, W.; Huang, C.-Y. Joint Sequence Learning and Cross-Modality Convolution for 3D Biomedical Segmentation. In Proceedings of the IEEE Conference on Computer Vision and Pattern Recognition, Honolulu, HI, USA, 22-25 July 2017; pp. 3739-3746. [CrossRef]

20. Setio, A.A.A.; Ciompi, F.; Litjens, G.; Gerke, P.; Jacobs, C.; van Riel, S.J.; Wille, M.M.W.; Naqibullah, M.; Sánchez, C.I.; van Ginneken, B. Pulmonary Nodule Detection in CT Images: False Positive Reduction Using Multi-View Convolutional Networks. IEEE Trans. Med. Imag. 2016, 35, 1160-1169. [CrossRef]

21. Guo, Z.; Li, X.; Huang, H.; Guo, N.; Li, Q. Medical image segmentation based on multi-modal convolutional neural network: Study on image fusion schemes. In Proceedings of the 2018 IEEE 15th International Symposium on Biomedical Imaging (ISBI), Washington, DC, USA, 4-7 April 2018; pp. 903-907. [CrossRef]

22. Yang, M.; Xiao, X.; Liu, Z.; Sun, L.; Guo, W.; Cui, L.; Sun, D.; Zhang, P.; Yang, G. Deep RetinaNet for Dynamic Left Ventricle Detection in Multiview Echocardiography Classification. Sci. Program. 2020, 2020, 7025403. [CrossRef]

23. Hu, S.; Gao, Y.; Niu, Z.; Jiang, Y.; Li, L.; Xiao, X.; Wang, M.; Fang, E.F.; Wade, M.-S.; Xia, J.; et al. Weakly supervised deep learning for covid-19 infection detection and classification from ct images. IEEE Access 2020, 8, 118869-118883. [CrossRef]

24. Wang, Y.; Yue, W.; Li, X.; Liu, S.; Guo, L.; Xu, H.; Zhang, H.; Yang, G. Comparison study of radiomics and deep learning-based methods for thyroid nodules classification using ultrasound images. IEEE Access 2020, 8, 52010-52017. [CrossRef]

25. Alzubaidi, L.; Zhang, J.; Humaidi, A.J.; Al-Dujaili, A.; Duan, Y.; Al-Shamma, O.; Santamaría, J.; Fadhel, M.A.; Al-Amidie, M.; Farhan, L. Review of deep learning: Concepts, CNN architectures, challenges, applications, future directions. J. Big Data 2021, 8, 1-74. [CrossRef] [PubMed]

26. Han, C.; Rundo, L.; Murao, K.; Noguchi, T.; Shimahara, Y.; Milacski, Z.Á.; Koshino, S.; Sala, E.; Nakayama, H.; Satoh, S. MADGAN: Unsupervised medical anomaly detection GAN using multiple adjacent brain MRI slice reconstruction. BMC Bioinform. 2021, 22, 039361. [CrossRef] [PubMed]

27. Liu, Y.; Yang, G.; Afshari, M.S.; Hosseiny, M.; Azadikhah, A.; Zhong, X.; Reiter, R.E.; Lee, Y.; Raman, S.S.; Sung, K. Automatic prostate zonal segmentation using fully convolutional network with feature pyramid attention. IEEE Access 2019, 7, 163626-163632. [CrossRef]

28. Wu, Y.; Suzan, H.; Diego, A.-Á.; Peter, G.; Li, B.; Gao, Y.; Firmin, D.; Keegan, J.; Yang, G. Fast and automated segmentation for the three-directional multi-slice cine myocardial velocity mapping. Diagnostics 2021, 11, 346. [CrossRef] [PubMed]

29. Yang, G.; Chen, J.; Gao, Z.; Li, S.; Ni, H.; Angelini, E.; Wong, T.; Mohiaddin, R.; Nyktari, E.; Wage, R.; et al. Simultaneous left atrium anatomy and scar segmentations via deep learning in multiview information with attention. Future Gener. Comput. Syst. 2020, 107, 215-228. [CrossRef]

30. Zhu, X.; Su, W.; Lu, L.; Li, B.; Wang, X.; Dai, J. Deformable DETR: Deformable Transformers for End-to-End Object Detection. arXiv 2020, arXiv:2010.04159.

31. Srinivas, A.; Lin, T.Y.; Parmar, N.; Shlens, J.; Abbeel, P.; Vaswani, A. Bottleneck transformers for visual recognition. In Proceedings of the IEEE/CVF Conference on Computer Vision and Pattern Recognition (CVPR), Seattle, WA, USA, 19-25 June 2021; pp. 16519-16529.

32. Chen, M.; Radford, A.; Child, R.; Wu, J.; Jun, H.; Luan, D.; Sutskever, I. Generative pretraining from pixels. In Proceedings of the International Conference on Machine Learning (ICML), Vienna, Austria, 13-18 July 2020; pp. 1691-1703.

33. Chen, J.; Lu, Y.; Yu, Q.; Luo, X.; Adeli, E.; Wang, Y.; Lu, L.; Yuille, A.L.; Zhou, Y. Transunet: Transformers Make Strong Encoders for Medical Image Segmentation. arXiv 2021, arXiv:2102.04306.

34. Xie, Y.; Zhang, J.; Shen, C.; Xia, Y. CoTr: Efficiently Bridging CNN and Transformer for 3D Medical Image Segmentation. arXiv 2021, arXiv:2103.03024.

35. Hatamizadeh, A.; Yang, D.; Roth, H.; Xu, D. Unetr: Transformers for 3D Medical Image Segmentation. arXiv 2021, arXiv:2103.10504.

36. He, K.; Zhang, X.; Ren, S.; Sun, J. Deep Residual Learning for Image Recognition. In Proceedings of the IEEE Conference on Computer Vision and Pattern Recognition (CVPR), Las Vegas, NV, USA, 26 June-1 July 2016; pp. 770-778.

37. Wang, X.; Girshick, R.; Gupta, A.; He, K. Non-Local Neural Networks. In Proceedings of the IEEE Conference on Computer Vision and Pattern Recognition (CVPR), Salt Lake City, UT, USA, 18-22 June 2018; pp. 7794-7803.

38. Hendrycks, D.; Gimpel, K. Gaussian Error Linear Units (GELUs). arXiv 2016, arXiv:1606.08415.

39. Liu, Y.; Yang, G.; Hosseiny, M.; Azadikhah, A.; Mirak, S.A.; Miao, Q.; Raman, S.S.; Sung, K. Exploring uncertainty measures in Bayesian deep attentive neural networks for prostate zonal segmentation. IEEE Access 2020, 8, 151817-151828. [CrossRef] 
40. Lima, R.A.; Tavares, M.R.; Dias, F.L.; Kligerman, J.; Nascimento, M.F.; Barbosa, M.M.; Cernea, C.R.; Soares, J.R.; Santos, I.C.; Salviano, S. Clinical Prognostic Factors in Malignant Parotid Gland Tumors. Otolaryngol. Neck Surg. 2005, 133, 702-708. [CrossRef]

41. Joe, V.Q.; Westesson, P.L. Tumors of the parotid gland: MR imaging characteristics of various histologic types. Am. J. Roentgenol. 1994, 163, 433-438. [CrossRef] [PubMed]

42. Rundo, L.; Militello, C.; Tangherloni, A.; Russo, G.; Vitabile, S.; Gilardi, M.C.; Mauri, G. NeXt for neuro-radiosurgery: A fully automatic approach for necrosis extraction in brain tumor MRI using an unsupervised machine learning technique. Int. J. Imaging Syst. Technol. 2018, 28, 21-37. [CrossRef]

43. van Dijk, L.V.; Thor, M.; Steenbakkers, R.J.H.M.; Apte, A.; Zhai, T.-T.; Borra, R.; Noordzij, W.; Estilo, C.; Lee, N.; Langendijk, J.A.; et al. Parotid gland fat related Magnetic Resonance image biomarkers improve prediction of late radiation-induced xerostomia. Radiother. Oncol. 2018, 128, 459-466. [CrossRef] [PubMed]

44. Jiang, J.-S.; Zhu, L.-N.; Chen, W.; Chen, L.; Su, G.-Y.; Xu, X.-Q.; Wu, F.-Y. Added value of susceptibility-weighted imaging to diffusion-weighted imaging in the characterization of parotid gland tumors. Eur. Arch. Otorhinolaryngol. 2020, 277, $2839-2846$. [CrossRef] [PubMed]

45. Otsu, N. A threshold selection method from gray-level histograms. IEEE Trans. Syst. Man. Cybern. 1979, 9, 62-66. [CrossRef]

46. Bien, N.; Rajpurkar, P.; Ball, R.L.; Irvin, J.; Park, A.; Jones, E.; Bereket, M.; Patel, B.N.; Yeom, K.W.; Shpanskaya, K.; et al. Deep-learning-assisted diagnosis for knee magnetic resonance imaging: Development and retrospective validation of MRNet. PLoS Med. 2018, 15, e1002699. [CrossRef] [PubMed]

47. Paszke, A.; Gross, S.; Massa, F.; Lerer, A.; Bradbury, J.; Chanan, G.; Killeen, T.; Lin, Z.; Gimelshein, N.; Antiga, L.; et al. PyTorch: An Imperative Style, High-Performance Deep Learning Library. arXiv 2019, arXiv:1912.0170.

48. Pérez-García, F.; Sparks, R.; Ourselin, S. TorchIO: A Python Library for Efficient Loading, Preprocessing, Augmentation and Patch-Based Sampling of Medical Images in Deep Learning. arXiv 2020, arXiv:2003.04696.

49. Qiu, Z.; Yao, T.; Mei, T. Learning Spatio-Temporal Representation With Pseudo-3D Residual Networks. In Proceedings of the IEEE International Conference on Computer Vision (ICCV), Venice, Italy, 22-29 October 2017; pp. 5533-5541.

50. Tran, D.; Bourdev, L.; Fergus, R.; Torresani, L.; Paluri, M. Learning Spatiotemporal Features with 3D Convolutional Networks. In Proceedings of the IEEE International Conference on Computer Vision (ICCV), Santiago, Chile, 13-16 December 2015; pp. 4489-4497.

51. Tsai, C.-H.; Kiryati, N.; Konen, E.; Eshed, I.; Mayer, A. Knee Injury Detection Using MRI with Efficiently-Layered Network (ELNet). Med. Imag. Deep Learn. 2020, 121, 784-794.

52. Dunnhofer, M.; Martinel, N.; Micheloni, C. Improving MRI-based Knee Disorder Diagnosis with Pyramidal Feature Details. Available online: https:/ / openreview.net/forum?id=7psPmlNffvg (accessed on 1 July 2021).

53. Krizhevsky, A.; Sutskever, I.; Hinton, G.E. ImageNet classification with deep convolutional neural networks. Adv. Neural Inf. Process. Syst. 2012, 25, 1097-1105. [CrossRef] 